\title{
Research on the Development Strategies of the Urban Fitness Coaches in Qingdao
}

\author{
$\mathrm{Li} \mathrm{Liu}^{1}$ \\ ${ }^{1}$ Department of Physical Education, Qingdao University of Science and Technology, Qingdao, China \\ Correspondence: Li Liu, Department of Physical Education, Qingdao University of Science and Technology, \\ Qingdao, China. E-mail: 13969851549@163.com
}

Received: April 27, 2014 Accepted: May 15, 2014 Online Published: May 30, 2014

doi:10.5539/ass.v10n11p225 URL: http://dx.doi.org/10.5539/ass.v10n11p225

\begin{abstract}
Basing on surveys on the current status of partially fitness club coaches, this paper makes a study of the operational state of them by means of documents, interviews, questionnaires, concept investigation and mathematical statistics. What's more, this paper applies sports marketing and other aspects of the sports industry knowledge with experts predicts to build a theoretical framework of our commercial fitness club management, aiming at providing theoretical support for the management of fitness club coaches to the unstained, stable and healthy development in Qingdao.
\end{abstract}

Keywords: fitness clubs, fitness coaches, development strategies

Since the reform and opening up, Chinese government attaches importance to the development of mass sports increasingly, effectively stimulating people's enthusiasm for physical exercise. Especially the success of the 2008 Beijing Olympic Games set off a wave of national sports fitness in the land of China. The needs of the physical fitness are rising. The "Twelfth Five-Year Plan" clearly states to promote rapid and coordinated development of mass sports, competitive sports, sports industry, constructing vigorous city in sports in Qingdao. The fitness coaches can even determine the number of members of the club in some way. There are 39,000 fitness trainers in China, and this number is growing at an annual growth rate of $15 \%$. Therefore, the level of fitness coaches plays a vital role in the fitness clubs.

Taking parts of fitness clubs in the urban area of Qingdao city as a breakthrough, this paper understands the development status of fitness coaches, studies their development model and problems, and explores development strategies to promote the orderly and healthy development of fitness coach teams and the sustained and stable development of the fitness clubs in the urban areas of Qingdao City.

\section{Study Objects and Research Methods}

\subsection{Study Objects}

This paper takes fitness coaches of clubs in Qingdao City downtown as the study objects

\subsection{Research Methods}

\subsubsection{Literature}

Since March 2013, we have inspected 32 related literatures of the fitness coach and fitness clubs in the library of Polytechnic University, College Reference Rooms and fitness centers in Qingdao. The literature discusses the Status and Development of athletic clubs and fitness coaches from different angles, and the current position and role of fitness trainers in the clubs. The literature provides an important theoretical support for the study of this paper.

\subsubsection{Interview}

Interviewing management workers and experts who have been engaged in fitness clubs for many years, we understand the status quo, trends and prospects of development of China's commercial sports fitness clubs, including problems of the management involved. Moreover, we grasp a great deal of valuable information and experience relating to the research. Our research has been recognized by experts. In addition, according to the research needs, I get some information on the operations of the club in the form of telephone interviews and informal discussion with the club's management staff. Our interviews include the club's size, grade, price, 
curriculum, coaches, the customers, the sales and trends and other issues of fitness club market. On this basis, we visited 11 clubs, e.g., Impulse Fitness, Tera Wellness (Qingdao Branch), British Guido fitness, health and other local influential fitness clubs. By communicating with them, we can feel their needs of the fitness activities. What's more, we discuss issues and trends of the current health club that provides a more detailed factual basis for our research with the club managers, supervisors and fitness coaches.

\subsubsection{Questionnaire}

According to the need of this study, we design student questionnaires by means of sports and social survey methods. The literature establishes theoretical basis and framework for the specific content of the questionnaire design that we get from the school library and reference library and the Internet. Thus, we design the questionnaire by making comprehensive analysis of all aspects of materials.

\section{Research Results and Analysis}

\subsection{The Basic Situation of Urban Fitness Trainers in Qingdao}

Since the first fitness club was established in Qingdao City in 2001, it has gradually become a regular upscale fitness club with ten years' development. However, many problems still exist with the development of society, changes in the market economy, as well as the improvement on health awareness, health awareness and increasing demand for health services. The problems are as follows (1) how to better manage modern clubs; (2) how to do a better job of technical services; (3) what problems still exist in the service process;(4) the status of the development of fitness club coaches, and characteristics (5) what problems still exist, and the growing trend (6) targeted measures to the management is of great significance to promote the healthy development of commercial sports fitness club.

\subsubsection{The Scale of Fitness Clubs}

The size of commercial sports fitness club not only directly reflects their investment costs, but also the factors that directly affect the coaches. The size should adapt the level of local economic development and people's consumption level and consumer' attitude, otherwise it will cause trouble to the management which directly affect the ultimate benefit of the clubs.

Table 1. List-scale of commercial operations fitness clubs

\begin{tabular}{lllll}
\hline Scale & Total Investment (Million) & Percentage $(\%)$ & Size $\left(\mathrm{m}^{2}\right)$ & Percentage $(\%)$ \\
\hline Small & $<=0.5$ & 27.08 & $<=500$ & 11.46 \\
Medium & $0.5-5$ & 35.42 & $500-1000$ & 55.21 \\
Large & $>5$ & 37.50 & $>1000$ & 33.33 \\
\hline
\end{tabular}

The scale of fitness club is divided into large, medium and small types according to the total investment and business area. Investment of 0.5 million, the operating area of 500 square meters is small; investment of 50-500 million and operating area of 500-1000 square meters is medium-sized; Investment of 5 million, the operating area in more than 1000 square meters is large. As can be seen from Table 3-1, the number of medium-sized health clubs is largest currently, followed by large health clubs, the number of small clubs is relatively small. In addition, from the survey, we can see small and medium-sized clubs are mostly located in Shibei district and Licang and other areas. On the contrary, large fitness club are mostly located in Shinan, such as YING PAISI, four stores in Qingdao, all located in Shinan and Laoshan District. Overall, the scale of operations in Qingdao are of varying sizes like others, showing the characteristics of diversification, the number of large and medium sized clubs is largest.

\subsubsection{Membership Conditions of Fitness Club}

The number of membership in fitness club directly involves the number of coaches and the vital interests of trainers. A well-run fitness club coaches should show the replacement of old and young. Marshals' coach, private teachers and group coaches joint each other to maintain members to improve the business of the club.

According to the survey, Ying Paisa, One trillion Wade, Ocean wide Elite are relatively large health clubs. Two of four Ying Paisa clubs locate in the city of Qingdao. There are more than 2,000 members in the clubs from registration. However, Ocean wide Elite is lack of the fitness trainers relatively. There is no way to lead members to do the fitness. Han Long leisure club was just registered last year. The club has adopted 388 
members in a year. the club develops in good condition. golf is a relatively trendy sport that is welcome in the high salaries. Club coaches are professionally trained to lead members participating in more than 30 games. Sunlight dynamic and Junlong Club are far from the city. The number of members is less; operating conditions need to be improved.

Comprehensively speaking, with the continuous improvement of fitness market and the development of people's health' awareness, and raise of people's income, personal trainers now is increasing from 2001 when the club only have marshals aerobics instructors. That is to say, personal trainer is one essential service in the club, but in a small club aerobics instructor takes a substantial portion, personal trainer followed. Pedal bicycles and equipment coaches account for a smaller proportion. Golf coach has higher skill level, focusing on specialized sites. Almighty coach is relatively lack. These problems will be solved with the development of fitness clubs and improvement of people's awareness gradually.

\subsubsection{Fees of Commercial Sports Fitness Club}

As it is shown in Figure 1, the vast majority of club takes the way of membership with fragmented system to get the charge. The number is $87.50 \%$, and the remaining $12.5 \%$ takes the way of membership to get the fees, without receiving scattered trainees. According to the survey, these clubs are all large-scale, high-end brands, such as Impulse, Tera and other fitness clubs, where consumers can not meet single fitness aspirations, but they can use a single time experience in the marketing vouches that the initial access to the fitness club or old members with new members to experience fitness, in order to promote consumption.

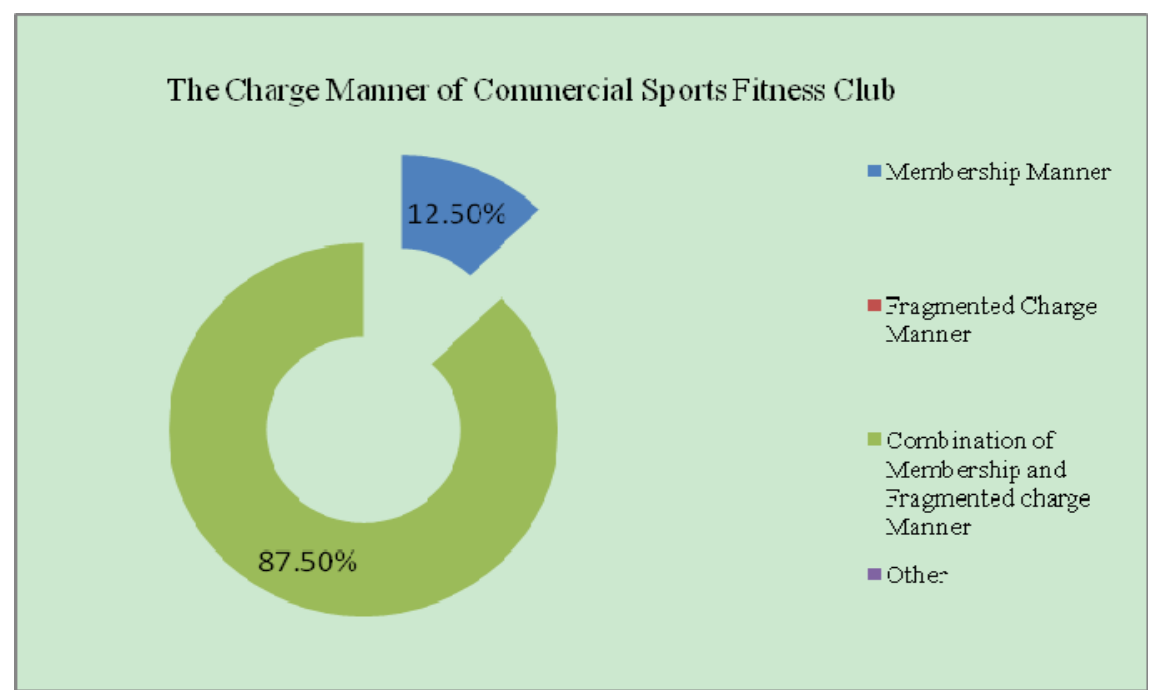

Figure 1. Charging methods of schematic fitness club

In terms of categories of membership cards, the vast majority of clubs are of diversity characteristics. In particular, some smaller, middle and low-level club has all kinds of cards, such as the second card, Day cards, monthly cards, season cards, half- a-year cards, and yearly cards. But some high-end clubs tend to exhibit the characteristics of the majority of long-term cards.

In terms of fees, different features are also showed, and even some disparities. According to the survey results, fees in 2000 Yuan are in most of the clubs, accounting for 52.08 percent. The lowest is under 1,000 Yuan, the highest in more than 3,000 Yuan. Middle and low annual fees are around 1500 at the club. It can be shown different characteristics of the annul fees due to the different levels of economic development, regional differences of people's income level. Studies have shown there is a big difference of annual fees in which the different regions of the same club in the same city, and the difference is very significant. For example Hall of Fuzhou Road and Guizhou Road Museum of Impulse Fitness, though they are in shinan, but in different regions, the price difference is also great. The income is the same for coaches. In high fees clubs and more private tasks, the coaches' income is significantly lower than the inferior regional clubs. Quite a few people not only stay on the basic functions "To Fitness", but gradually take fitness as a lifestyle, an indispensable element of life. The fitness club operators should take full account of the level of economic development in the region, increasing management of coaches, strengthen professional training in order to win more member support. 


\subsection{Structural Condition of Fitness Instructor Teams}

\subsubsection{Structure Type of Gender and Age}

Table 2. Structure type of gender and age

\begin{tabular}{lll}
\hline & Frequency & Percentage \\
\hline Gender & & \\
Male & 60 & $55 \%$ \\
Female & 50 & $45 \%$ \\
\hline Age & & \\
\hline$<=20$ & 15 & $13.7 \%$ \\
$20-30$ & 75 & $68.1 \%$ \\
$>30$ & 20 & $18.2 \%$ \\
\hline
\end{tabular}

From Table 2, we can see the number of males is bigger than that of females in urban of Qingdao, $55 \%$ males, females accounted for $45 \%$. As the fitness trainer shows masculinity in the work force, making the most members prefer male coaches that possess these qualities, while women are mostly good at types of courses like aerobics and yoga. Therefore fitness club prefer male coaches. In age distribution, the majority is concentrated in the 20 to 30 years old, accounting for $68.1 \%$ that plays mainstay role in the whole coaching team. There is no doubt that the younger coach also brings vitality and passion to the team for the fitness industry as a whole, young and energetic, vibrant, receptive to new things, new exercise program for emerging timely and grasp accurate requirements, so the club and members of this age group fitness instructor comparison welcome, but also very trust conducive to the development of the urban area of Qingdao City fitness career.

\subsubsection{Academic Structure}

Table 3. Situation of academic structure

\begin{tabular}{lll}
\hline Education Level & Frequency & Percentage \\
\hline Junior High School Degree & 0 & 0 \\
High School Education Degree & 12 & $10.9 \%$ \\
College Degree & 42 & $38.2 \%$ \\
Bachelor's Degree & 36 & $32.7 \%$ \\
Graduate Degrees & 20 & $18.2 \%$ \\
\hline
\end{tabular}

Academic qualifications is one of the important criterias to measure a person's level of knowledge and service quality, so the level of educational structure reflects the growth potential and change of the fitness' industry to some extent. The survey results show that Qingdao City urban center fitness instructor's educational background is mediate, mainly junior college and university, accounting for $70.9 \%$. Although the education level is above the average level, but mostly is part-based, many people are not professionally trained and being technical educated. To some certain extent, it also hampers the development of the region's fitness clubs. As we all know, a qualified fitness trainer can not only give members some guidance on fitness classes, but must have a deeper understanding and awareness of physiology, anatomy, nutrition and other related disciplines, taking higher education as a support. Generally speaking, a highly educated fitness trainer can better promote the development of a fitness club to ensure scientific of fitness guidance.

\subsubsection{Qualification Structure}

Fitness coach is now very common in the island city, but a personal trainer is not well acquainted with. Via investigation on personal fitness trainers, we can learn that only almost 200 Castle personal trainers are certified. They are mostly employed by business owners, real estate executives, companies and other wealthy middle-level cadres. Survey shows that, except Impulse, other health clubs are "undocumented private education". Most of them are young people. They do part-time job at the club with no qualifications. The recruitment is very strict in 
Impulse, not only pass professional knowledge test, but also a three month trial period. "Knowledge does not necessarily know how to teach well, the key is to see the actual operation, interaction and communication between students". Management system of Impulse is more standard compared to several other health clubs. The undocumented private education is not existed.

Personal fitness trainer has four or five decades of history in foreign countries. It also develops over the years in Beijing, Shanghai and other cities. While in the island city, personal trainer appears for nearly a decade. The pace of development of private education industry in Qingdao in recent years can be described as the word of "blowout". Through field investigation, it is a must to be a personal trainer with national vocational qualifications and coaching certificate. In recent years, with the rapid development of the fitness industry, the demand for fitness instructors have gradually increased, so the variety of professional coaches qualified training institutions are also increasing. However, professional fitness club in the urban area of Qingdao city starts late, accredited training institutions is lack. Some fitness clubs introduce training institutions for the qualification and training of the coaches, but still in its infancy. The scale of the trainees, teachers and certification examinations are highly irregular, with no credibility. Thus, each health club should not only work together to establish certification training institutions to adapt to the development of the club in the competition but also make the training curriculum more scientific, systematic and complete, taking basic theory and knowledge of learning, skills training as the starting point and proper methods fitness trainer should have to increase the usefulness and timeliness of training. Take the professional skills and certificates as a measure to weigh the level of fitness club, otherwise bound to affect the development of the team in the urban area of Qingdao City.

\subsubsection{Source of Fitness Trainer}

Table 4. Source of fitness trainer

\begin{tabular}{lll}
\hline Source & Frequency & Percentage \\
\hline Full-time Graduated Students & 44 & $40 \%$ \\
Part-time & 58 & $53 \%$ \\
Other & 8 & $7 \%$ \\
\hline
\end{tabular}

At present, the source of fitness coaches includes part-time fitness instructor (including part-time employees, part-time college students), related professional graduates directly into the health club as a fitness coach, as well as other sources in Qingdao City. Table 4 shows, 58\% are part-time staff in the health clubs, most of them have their professional expertise, mastering a high level of professional skill with some teaching methods to make the crowd master their favorite and appropriate fitness classes. Because these coaches have their own formal occupation, lacking more effort to make their own curriculum diversified, not timely and effective contacts the latest fitness programs. Fitness members will feel boring in the long process of teaching. $40 \%$ of the fitness trainer is composed by the relevant graduates. They not only have a certain expertise, but also in the full process of enriching themselves, enrich their knowledge of all aspects of fitness to grasp and develop new projects, more easily toward all-around development. Almighty coach development is conducive to the development of a health club. But with no fixed source of talent, to some extent, these coaches are relatively lack in Qingdao, which restricts the further development of the health club. Thus, full-time personnel are few in the fitness industry that can not meet the requirements of the fitness club.

\subsection{Fitness Trainer Work}

\subsubsection{Guide Content}

A good trainer should guide his members in different ways. Especially for the private trainer, he not only provides the ordinary exercises for members, such as how to operate the applications, but also assesses the condition of the bodies for members timely, such as compare the changes of weight, height, blood pressure, skin fold thickness after the exercises. Above all, the private trainer should provide private personal suggestions and diet lists for members. It is known that there are only about 200 private trainers with certificates in Qingdao, mostly employed by the rich man, such as corporate owners, company elites, which make them get closed to be a millionaire. But it is not easy to be a personal trainer. They should not only help clients fitness, but also as a private nanny in peacetime. Customer's diet, living habits should be recorded. They should know something of nutrition and anatomy. Which piece of customer's muscle needs to be exercised should be clear. Taking over a new student, the first step is to let him/her to do a special fitness test in Impulse. The main contents include 
detection of muscle strength, endurance and cardio endurance. Based on the member's fitness test, indicator assesses their body. To assess the role is to further understand the situation of students to make more reasonable arrangements of different exercise for students.

"Is there much usual socializing?" "Eat more meat, or vegetables?" Including physical testing, personal trainer will do a detailed questionnaire about life, eating area for new students in Impulse. This is another advantage of private coach compared to ordinary one. Now the latest fitness concept is emphasized on reduced-fat rather than the weight loss. Private Coach is not only a good fitness coach, but also be a "private nutritionist". Therefore, it is necessary for participants to make reasonable adjustments of lifestyle habits, especially diet to couple with regular exercise. Only by this can let the fat within the body slowly lose. Personal trainer here all has an iPad, which records the information and results of each trainee. "This is equivalent to sports electronic files of members, students will have their own name and password, just log in through the website, you can clearly see his physical condition".

Personal trainers are fewer and develop slower in other fitness club in Qingdao, mostly popular coach. They mainly teach fitness subjects, lack of guidance of comprehensive index, lack of comprehensive, integrated health prescriptions, professional and technical single, which is not conducive to the orderly development of a comprehensive fitness coach.

\subsubsection{The Size of the Crowd Guidance}

With the growing awareness of people's health, the number of population involved in commercial fitness activities increases. From Table 5, we can see main course in each club are aerobics classes, cycling classes, pedal courses, equipment Exercise and a few golf courses, lacking innovation and other factors restricting the number of members participating in the course of practice. Moreover, curriculum is few, mainly due to their expertise scarcity. No innovative talents, no innovative courses. Although the number is increasing in fitness membership, the above constraint makes the population size small, tends to a fixed value. It not only restricts the development of a health club, but also influence and restrict self-improvement of a fitness trainer to some extent.

\subsubsection{The Ability to Structure}

Table 5. Fitness trainer statistics ability cognition

\begin{tabular}{lll}
\hline Abilities & Frequency & Percentage \\
\hline Communication & 32 & $29 \%$ \\
Teaching & 22 & $20 \%$ \\
Learning & 16 & $15 \%$ \\
Innovation & 8 & $7 \%$ \\
Sociability & 12 & $11 \%$ \\
Collaboration & 6 & $5 \%$ \\
Planning & 14 & $13 \%$ \\
\hline
\end{tabular}

As can be seen from Table 5, as a qualified trainer, he should have a wide range of capabilities. From the survey, we found that $29 \%$ of the coaches suppose that possess the communication ability is very important to sell yourself, which also would get the recognition and support of your members, and this would be benefit to establish the prestige for coaches. In the teaching process, the coaches meet the requirements and interests of their members and adjust structure of the course would effectively improve the effect of training. Also, we can got the information that teaching ability, learning ability and planning ability are also occupy a higher proportion of coaches, which make up more than $48 \%$, however, we found that the coaches take less emphasis on innovation ability, sociability and collaboration through the investigation, which in turn are essential for a coach trainer. Strengthen these aspects of training not only improve the quality of coaches but the needs for the rapid development of the fitness industry. In order to be able to promptly and effectively resolve problems during fitness instruction, coaches must constantly update their knowledge on innovation and collaborative. 


\subsubsection{Analysis of the Situation of Scientific Guidance}

Table 6. Analysis of the situation of scientific guidance

\begin{tabular}{llll}
\hline & Outstanding & Normal & Bad \\
\hline Scientific guidance & 62 & 43 & 5 \\
Formulate different training methods according to different members & 32 & 60 & 18 \\
Theoretical analysis on training methods & 31 & 72 & 7 \\
Answer related questions with professional knowledge & 29 & 77 & 4 \\
\hline
\end{tabular}

Table 6 shows that the vast majority of fitness coaches consider themselves scientific guidance capabilities are not very good and only few abilities are outstanding. This may because the education of overall fitness coach is just at the middle level for the urban area of Qingdao city, and most of coaches are part-time coaches, which low down the teaching level and effect. Therefore, whether possess a professional knowledge and technology directly affect the training results. Nowadays, with the increasing variety of fitness classes more and more popular, coaches acquire more and more relevant expertise for training, which leads a considerable part of them improve their confidence and satisfaction in the process of guide. We can draw from this table that coaches only to master fitness skill and put theoretical knowledge into teaching practice, they can provide comprehensively and scientifically guide for their members.

\subsection{Social Conditions of Fitness Trainer}

\subsubsection{Case of Wages}

Table 7. Case of wages

\begin{tabular}{llll}
\hline Class fees (yuan) & $<=50$ & $50-100$ & $>100$ \\
\hline Number of Members & 10 & 65 & 35 \\
Percentage & $9 \%$ & $59 \%$ & $32 \%$ \\
\hline
\end{tabular}

As it can be seen from Table 2-4-1, 59\% of fee of coach class is between 50-100 Yuan, mainly in the form of class fee with no fixed monthly salary. Compared with other industries, their wages are in the low ground. Although the awareness of people has changed to spend more money on health, they are affected by inconvenience of traffic, small-scale of fitness areas and other factors; resulting non-substantial benefit of the health club, thus further affecting the salary status of these health club fitness instructor overall low. However, the salary of personal trainer is relatively high, basically a hundred in an hour. In fact, the income gap is quite big between personal trainers. Personal wages is basically composed of basic salary and commission that is mainly determined by the number of hours of each person. Although commission is different in each club, the lack of the well-known personal trainer make them can easily gain 10,000 Yuan a month. Compared to ordinary style of members, cost of private coach is certainly higher. Thus, personal trainer fitness is mainly invited by those who have a certain economic base. Among the members, a personal trainer is hired by business owners and middle-level cadres. Some ordinary workers opt for private coaching because they know the role of fitness and quickly adjust the physical condition, will.

\section{Conclusions and Recommendations}

\subsection{Conclusions}

- Firstly, although there are many health clubs in the urban area of Qingdao city, but the scale is smaller and lack of all-around coaches. Secondly, the fitness classes are single. Thirdly, part-time coaches are more than the proportion of the full-time. Most of team are young, and their educational level is not overall high. What's more, qualified teachers are fewer, and there are no professional fitness instructor certification bodies. Thus, the fitness trainer industry has a broad space for development.

- There are not comprehensive and rational content of the fitness coaching work. First of all, the guiding crowd is small. Secondly, scientific guidance situation is boring. Moreover, the curriculum is lack of innovation. Furthermore, the crowd's favorite fitness programs can not be fully grasped in this region. 
- The average wages of fitness coaches is between 50-100 yuan (each class fee) in the urban area of Qingdao City. The social security system needs to be improved, and the social role of fitness trainer is difficult to get adequate positioning on the impact of regional factors.

- The fitness market is not standardized. All of the investors want to get good returns in the short term. Health investment is a lifelong philosophy, but market positioning of a lot of gyms is not accurate. However, the follow-up services for members can not keep up after they open the membership cards, thus members' interests suffer a lot.

- Nowadays, fitness content has a wide range. In addition to the sports, fitness equipment includes a variety of dance classes and yoga classes parade and outdoor golf courses. What's more, it also includes medical rehabilitation, massage and health management and so on. But now fitness equipment is common in urban fitness areas.

\subsection{Recommendations}

- It should strengthen the construction of fitness instructor team and personnel training. To address the current lack of fitness trainers, fitness knowledge and training should be conducted on the existing coaches. They should constantly improve themselves at the same time. Fitness club can keep contact with the relevant effective authorities timely. The various seminars and training courses can be organized regularly within the scope of improving the theory and practice level of coaches to bring up a highly qualified team of professionals to adapt to the development of the clubs.

- $\quad$ Establish and improve the incentive and competitive mechanism. Clubs and coaches should pay attention to the improvement of their qualities to actively create opportunities for further study and grade promotion, guarantee professional qualities of fitness trainers. The club's economic benefits are closely linked to coaches' income. The coaches' work, qualifications, and performance decide their salary grades. Considering the long-term interests of the health club, we should focuses on the development of all staff.

- Establish investigated monitoring mechanisms of fitness coaches, and conduct stage inspection, supervision, and assessment of coaches to develop their practitioner level.

- $\quad$ Establish a sound social security system of fitness trainers. Local authorities and the club should be based on its own conditions, strengthen internal management and reform, and introduce appropriate standard wages and social security measures to fully protect the fitness trainer industry regularization. Fitness club should take into account of the ability to enhance each coach while expanding services, identify social role of coaches, thereby improve the quality of services.

\section{References}

Du, X. R., \& Gao, Y. H. (2005). Current Situation and Analysis high jade flower. In Beijing, Shanghai part of a health club personal trainer. Shanghai Sports Institute, 12(4), 32-34.

Qin, C. L. (2003). Organization and management of physical fitness activities (pp. 130-134). Beijing Sports University Press.

Wang, Y. (2005). Investigation and analysis of Nanjing large fitness club personal trainer status quo. Nanjing Institute of Physical Education, (3).

Xiang, J. H. (2007). Personal Trainer Career Design (pp. 45-47). Beijing: Beijing Sports University Press.

Zhang et al. (2002). The development of Chinese sports fitness club overview of. Beijing Sport University Newspaper, 26(6), 744-745.

Zhang, Y., \& Liu, B. (2000). Status quo and problems in our fitness entertainment market. Sport and Science, 26(2), 101-103.

\section{Copyrights}

Copyright for this article is retained by the author(s), with first publication rights granted to the journal.

This is an open-access article distributed under the terms and conditions of the Creative Commons Attribution license (http://creativecommons.org/licenses/by/3.0/). 